Women: 574,679 (114,936 cases, 459,743 controls (279,905 only negative tests, 179,838 never tested)), contributing 1,807,618 tests and 20,912 reproductive outcomes;

Men: 325,337 (65,068 cases, 260,269 controls) contributing 291,655 tests and 9,698 reproductive outcomes.

Conclusion This large, rich dataset including long term follow up of a nationally representative sample of men and women from Denmark is a step forward in addressing some of the outstanding questions about the population impact of chlamydia screening.

\section{P08.38 COST-EFFECTIVENESS OF CHLAMYDIA TESTING IN SCOTLAND}

${ }^{1} \mathrm{KJ}$ Looker, ${ }^{2} \mathrm{~L}$ Wallace, ${ }^{3} \mathrm{KME}$ Turner*. ${ }^{1}$ School of Social \& Community Medicine, University of Bristol, UK; ${ }^{2}$ Health Protection Scotland, Glasgow, UK; ${ }^{3}$ School of Veterinary Science, University of Bristol, UK

\subsection{6/sextrans-2015-052270.384}

Background Scottish chlamydia testing guidelines target symptomatic and high-risk asymptomatic individuals. Recent publications, indicating a low risk of progression to serious chlamydiarelated outcomes, particularly tubal factor infertility (TFI), question the validity of high levels of opportunistic testing especially among asymptomatic individuals.

Aim (s)/objectives To examine cost-effectiveness of current chlamydia testing to prevent TFI among those aged 15-24 in Scotland using cost per Quality-Adjusted Life Years (QALYs) gained and to consider alternative testing strategies.

Methods A compartmental deterministic model of chlamydia infection in those aged 15-24 in Scotland was developed to examine the impact of testing coverage and partner notification (PN) on number and cost of TFI cases prevented. Cost-effectiveness calculations were informed by best estimates of the QALYs lost due to TFI.

Results At 16.8\% baseline testing coverage (laboratory data), $4.4 \%$ prevalence (NATSAL-3) and assumed PN rate of 0.4 , the total testing cost is $£ 5.4$ million. This is estimated to prevent 258 TFI cases each year in young women. The cost per QALY gained is $£ 40,034$ compared with no testing, using a mid-range health state utility value (HSUV) for TFI $(0.76( \pm 0.24))$ and PID $(0.9( \pm 0.22))$. A $50 \%$ reduction in current testing would result in higher chlamydia prevalence and 84 more TFI cases.

Discussion/conclusion Current chlamydia testing activities in Scotland do not appear cost-effective. However, the model is sensitive to several parameters, particularly the HSUV and there are uncertainties in the current testing costs and progression to serious sequelae. There appears potential to improve chlamydia testing cost-effectiveness by increasing PN.

\section{P09 - Bacterial and other curable STI}

\section{P09.01 COST-EFFECTIVENESS OF TESTING FOR TRICHOMONAS VAGINALIS IN GENITOURINARY MEDICINE CLINICS AND PRIMARY CARE IN ENGLAND USING APTIMA TV NAAT}

${ }^{1} \mathrm{~K}$ Turner*, ${ }^{2} \mathrm{~J}$ Nicholls*, ${ }^{3} \mathrm{P}$ Muir, ${ }^{3} \mathrm{P}$ North, ${ }^{3} \mathrm{R}$ Ferguson, ${ }^{4} \mathrm{M}$ May, ${ }^{4} \mathrm{~J}$ Macleod, ${ }^{2,4} \mathrm{P}$ Horner. ${ }^{1}$ School of Veterinary Sciences, University of Bristol, Bristol, UK; ${ }^{2}$ Bristol Sexual Health Centre, University Hospitals Bristol NHS Trust, Bristol, UK; ${ }^{3}$ Public Health Laboratory Bristol, Public Health England, Bristol, UK; ${ }^{4}$ School of Social and Community Medicine, University of Bristol, Bristol, UK

\subsection{6/sextrans-2015-052270.385}

Background Laboratory tests for Trichomonas vaginalis (TV) using culture and microscopy in current practice have low sensitivity. However new, highly sensitive PCR-based nucleic acid amplification tests (TV NAATs) have been approved e.g. Aptima TV NAAT. It is not known how to optimally deploy these new tests.

Objectives To assess the cost-effectiveness of new TV NAAT tests for the diagnosis of TV infection in women attending genitourinary medicine (GUM) and primary care clinics in England. To inform decision-making about who should be offered TV testing.

Methods We analysed data from TV tests in residual chlamydia/ gonorrhoea samples from 9,000 women. We conducted notes review in GUM clinics to understand current management. We calculated the cost of testing for TV and the cost per additional case detected.

Results The cost of TV test as a new standalone test is $£ 15.19$, or $£ 7.62$ as an add-on to chlamydia/gonorrhoea NAAT tests. Microscopy/wet prep costs $£ 7.93$. Current testing detected only 9/50 GUM and 15/126 primary care cases identified using TV NAAT tests. The crude cost of adding TV to all chlamydia/gonorrhoea NAAT tests and removing microscopy, is $£ 291$ per additional case ( $£ 139$ per symptomatic and $£ 496$ per asymptomatic). The cost-effectiveness is sensitive to the underlying positivity (decreases within increasing positivity) and the differences between the amount and sensitivity of current testing practice.

Discussion/conclusion TV NAAT tests detected many more infections than current testing practice. If TV NAAT is added to current chlamydia/gonorrhoea testing in English GUM clinics, this would translate to an increase from 6,000 TV cases to 23,400 cases annually. The implications are that testing symptomatic women in GUM and possibly primary care for TV would be beneficial as positivity is similar to chlamydia in these groups. For asymptomatic women a more targeted approach may be more appropriate according to local demographics.

Disclosure of interest statement Hologic provided the tests for the Aptima TV NAAT research study and have sponsored the authors to present this data at ISSTDR. 